\title{
Как избежать одновременного открытия ключей в синхронных понижающих преобразователях при замедлении фронтов импульсов
}

\author{
$\Phi$. Досталь $^{1}$
}

УДК 621.314.1| ВАК 05.27.01

\begin{abstract}
Импульсные стабилизаторы завоевали широкую популярность у разработчиков источников питания постоянного тока, поскольку обеспечивают высокий КПд и отличаются гибкостью применения - на их основе можно создать различные топологии: повышающие, понижающие, инвертирующие. Высокая скорость нарастания сигналов коммутации в импульсных стабилизаторах обеспечивает значительное снижение потерь на переключение. Однако уменьшение фронтов импульсов влечет за собой ряд проблем. В диапазоне частоты коммутации примерно от 20 до 200 МГц резко возрастают помехи. Поэтому разработчик импульсных источников питания вынужден искать баланс между высоким КПд и низким уровнем помех в диапазоне высоких частот. Компания Analog Devices предлагает решение, которое позволяет снизить риск короткого замыкания в синхронных понижающих преобразователях при замедлении фронтов импульсов с целью уменьшения электромагнитных помех.
\end{abstract}

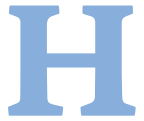

а рис. 1 показаны импульсы с высокой и низкой скоростью нарастания напряжения. Быстрые фронты сигналов вызывают интенсивное наведение помех на соседние участки схемы. Проводники печатных плат с быстроменяющимися сигналами посредством емкостной связи оказывают влияние на соседние линии с высоким полным сопротивлением. Проводники, в которых быстро изменяется ток, индуктивно связаны с соседними линиями печатной платы и наводят на них помехи. Если снизить скорость нарастания импульсов, можно минимизировать эти эффекты.

На рис. 2 представлена апробированная методика для асинхронных импульсных стабилизаторов. Как видно на схеме, один из двух ключей выполнен в виде диода Шоттки. Резистор, последовательно включенный с компенсационным конденсатором в цепи обратной связи $C_{\text {воот, }}$ через который подается напряжение на затвор п-канального MOSFET верхнего плеча, служит для замедления импульсов, коммутирующих ключ. Такой подход используется в интегрированных импульсных стабилизаторах, в которых нет непосредственного соединения с цепью затвора силового MOSFET. При использовании контроллера переключения с внешним MOSFET резистор также можно

Компания Analog Devices, инженер по применению,

frederik.dostal@analog.com включить в цепь затвора. Обычно применяют резистор сопротивлением менее 100 Ом.

Однако самые современные импульсные стабилизаторы - это синхронные стабилизаторы с активными ключами верхнего и нижнего плеча. В них нельзя замедлить нарастание импульсов с помощью резистора в цепи конденсатора $C_{\text {воот. }}$ Если в такой схеме использовать

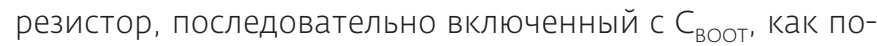
казано на рис. 3, переключение верхнего ключа также

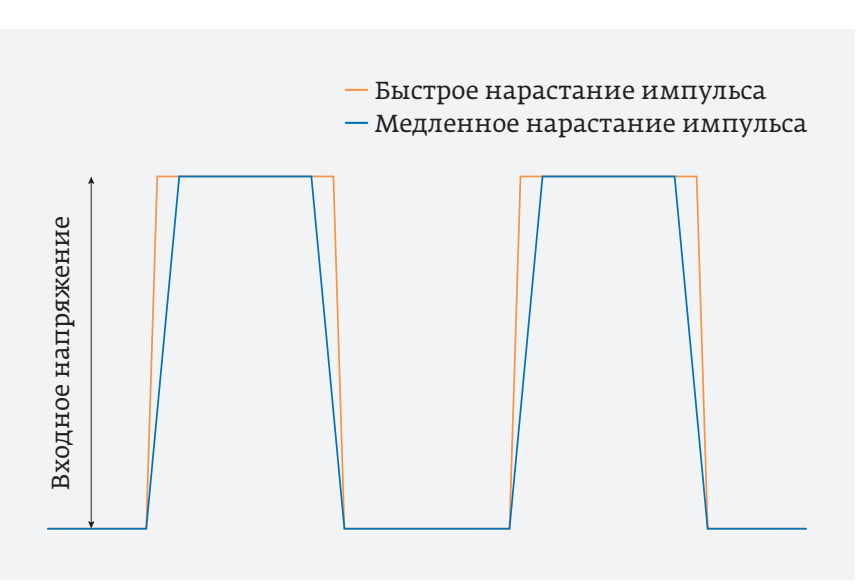

Рис. 1. Форма сигналов коммутации с разной длительностью фронта в импульсных источниках питания 


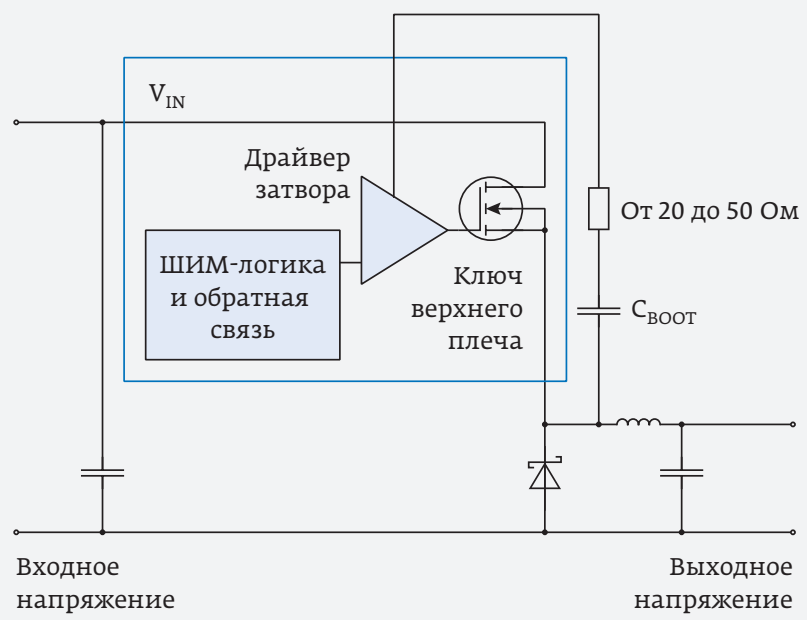

Рис. 2. Снижение скорости нарастания импульсов в асинхронном понижающем преобразователе с компенсационным конденсатором в цепи обратной связи

замедлится. Однако это может привести к тому, что ключ нижнего плеча не будет полностью выключен. Таким образом, может возникнуть ситуация, когда ключи как верхнего, так и нижнего плеча будут какое-то время одновременно включены, что может вызвать короткое замыкание входного напряжения на землю. Кроме того, на скорость нарастания импульсов также влияют такие факторы, как рабочая температура и нестабильность технологических процессов. Поэтому даже при тестировании в лабораторных условиях нельзя гарантировать безопасность работы этих устройств.

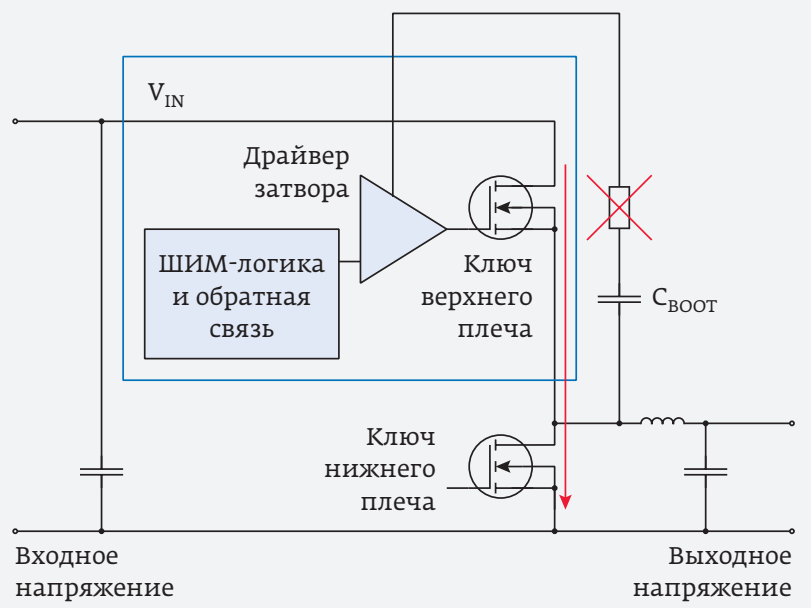

Рис. 3. Замедление нарастания импульсов в синхронных понижающих преобразователях может привести к короткому замыканию
ADP5014 - это четыре импульсных понижающих стабилизатора с низким уровнем шумов в 40-выводном корпусе LFCSP. Каждый канал этой микросхемы содержит мощные MOSFET в качестве ключей верхнего и нижнего плеча. Каналы можно запрограммировать на выходные токи от 1 до 4 А, реализована также возможность параллельного включения двух каналов, чтобы обеспечить ток на выходе до 8 A.

B ADP5014 предусмотрены два режима запуска. B ручном режиме доступно четыре прецизионных вывода разрешения для запуска каждого стабилизатора вручную. В режиме последовательного включения предусмотрен один групповой сигнал разрешения с программируемыми таймерами задержки включения и отключения по каждой шине питания. Частоту коммутации ADP5014 можно запрограммировать или синхронизировать от внешнего тактового сигнала в диапазоне от 500 кГц до 2,5 МГц.

К другим ключевым функциям устройства относятся ШИМ-режим, режим пониженного потребления, предупреждение о падении напряжения, активный разряд на выходе и флаг корректного уровня питания. Устройства оснащены всеми типами защит: блокировка питания при пониженном напряжении, защита от перегрузки по напряжению и току, защитное отключение при перегреве.

Ключевые особенности ADP5014:

- диапазон входных напряжений: от 2,75 до 6,0 В;

- программируемый диапазон выходного напряжения: от 0,5 В до 0,9 $\mathrm{PV}_{\mathrm{INX}}$;

- низкий уровень шумов на выходе:

$\sim 25 \mathrm{MkB}$ (rms) при $\mathrm{V}_{\text {OUT }} \leq \mathrm{V}_{\mathrm{REF}}$;

- точность на выходе $\pm 1,0 \%$ во всем диапазоне температур;

- выходной ток: до 4 А (до 8 А при параллельном включении двух каналов);

- регулируемая частота коммутации: от 500 кГц до 2,5 МГц;

- прецизионный вход разрешения с порогом 0,6 B;

- прецизионный компаратор контроля падения напряжения;

- активный ключ разряда выхода;

- ручной режим и режим последовательного включения;

- флаг корректного уровня питания;

- температура перехода: -40 ...125 ${ }^{\circ} \mathrm{C}$. 
Для замедления нарастания импульсов в синхронных импульсных стабилизаторах с интегрированными ключами следует использовать устройства, в которых можно установить крутизну фронтов импульсов с помощью встроенных схем. Пример такого устройства - ADP5014 от компании Analog Devices. В этой микросхеме реализовано решение, которое гарантирует, что при уменьшении скорости нарастания импульсов оба ключа не будут одновременно проводить ток, следовательно, не может возникнуть короткое замыкание. Причем это не требует применения резистора в цепи компенсационного конденсатора.

Что касается проблемы быстрых фронтов переключения, то нельзя не учитывать инновации последних лет.
Технология Silent Switcher от компании Analog Devices позволяет значительно (до 40 дБ, или в 10 тыс. раз) снизить электромагнитные помехи из-за высокой скорости нарастания импульсов. Это дает возможность разрабатывать импульсные источники питания с чрезвычайно малыми фронтами сигналов, отвечающие высоким требованиям по электромагнитной совместимости. Устройства семейства Silent Switcher в большинстве случаев позволяют избежать уменьшения скорости нарастания импульсов для снижения уровня электромагнитных помех. Благодаря этой технологии можно отказаться от поиска компромисса между достижением максимальной эффективности преобразования и минимальными генерируемыми помехами.

\section{КНИГИ ИЗДАТЕЛЬСТВА «ТЕХНОСФЕРА»}

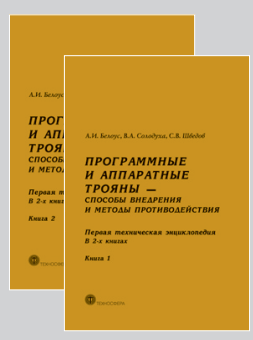

Цена за две книги 2400 руб.

\section{ПРОГРАММНЫЕ И АППАРАТНЫЕ} ТРОЯНЫ - СПОСОБЫ ВНЕДРЕНИЯ И МЕТОДЫ ПРОТИВОДЕЙСТВИЯ. ПЕРВАЯ ТЕХНИЧЕСКАЯ ЭНЦИКЛОПЕДИЯ

\author{
В 2-Х книгах
}

\author{
Белоус А. И., Солодуха В. А., Шведов С. В. \\ Под общей редакцией Белоуса А. И.
}

В двухтомнике исследован феномен программных и аппаратных троянов, которые фактически являются технологической платформой современного и перспективного кибероружия. В первой вводной главе показано, что развитие всех "обычных" и "новейших" видов вооружений дошло до такой стадии, что их использование на практике будет равносильно самоубийству начавшей войну стороны. Осознание этого факта привело к развитию информационно-технического оружия (кибероружия и нейрооружия). В последующих главах детально исследованы концепции, методы и примеры реализации этого вида оружия. Рассмотрены основные виды программных троянов, вирусов и шпионских программ, показан эволюционный путь развития аппаратных троянов от "ящиков" и "коробочек" до микросхем.

Книга ориентирована на специалистов по информационной безопасности, а также будет полезна всем интересующимся данной темой. 


\section{ANALOG
DEVICES}

AHEAD OF WHAT'S POSSIBLE'

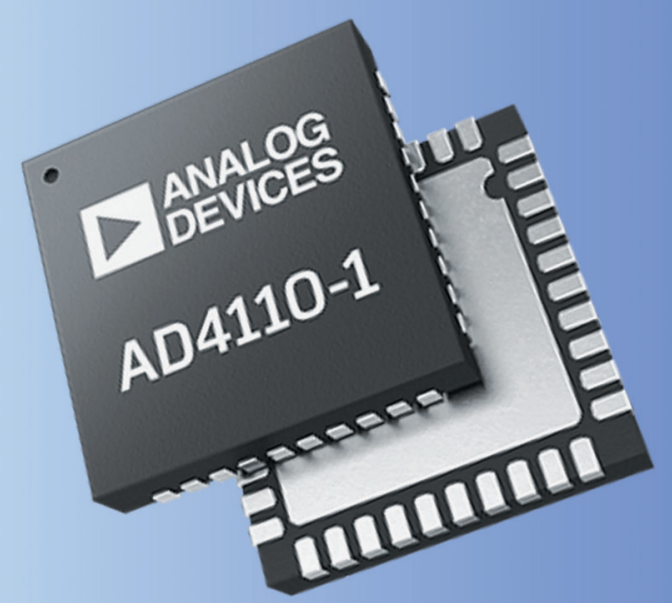

\section{УНИВЕРСАЛЬНЫЙ АЦП ДЛЯ КОНТРОЛЯ \\ ПРОИЗВОДСТВЕННЫХ ПРОЦЕССОВ}

ПРЕЦИЗИОННЫЙ 24-РАЗРЯДНЫЙ г-А АЦП ТРИ ТИПА ИЗМЕРЕНИЙ:

- Входное напряжение \pm 10 В

- Входной ток \pm 20 мА

- Температура

ВСТРОЕННАЯ ДИАГНОСТИКА И ЗАЩИТА

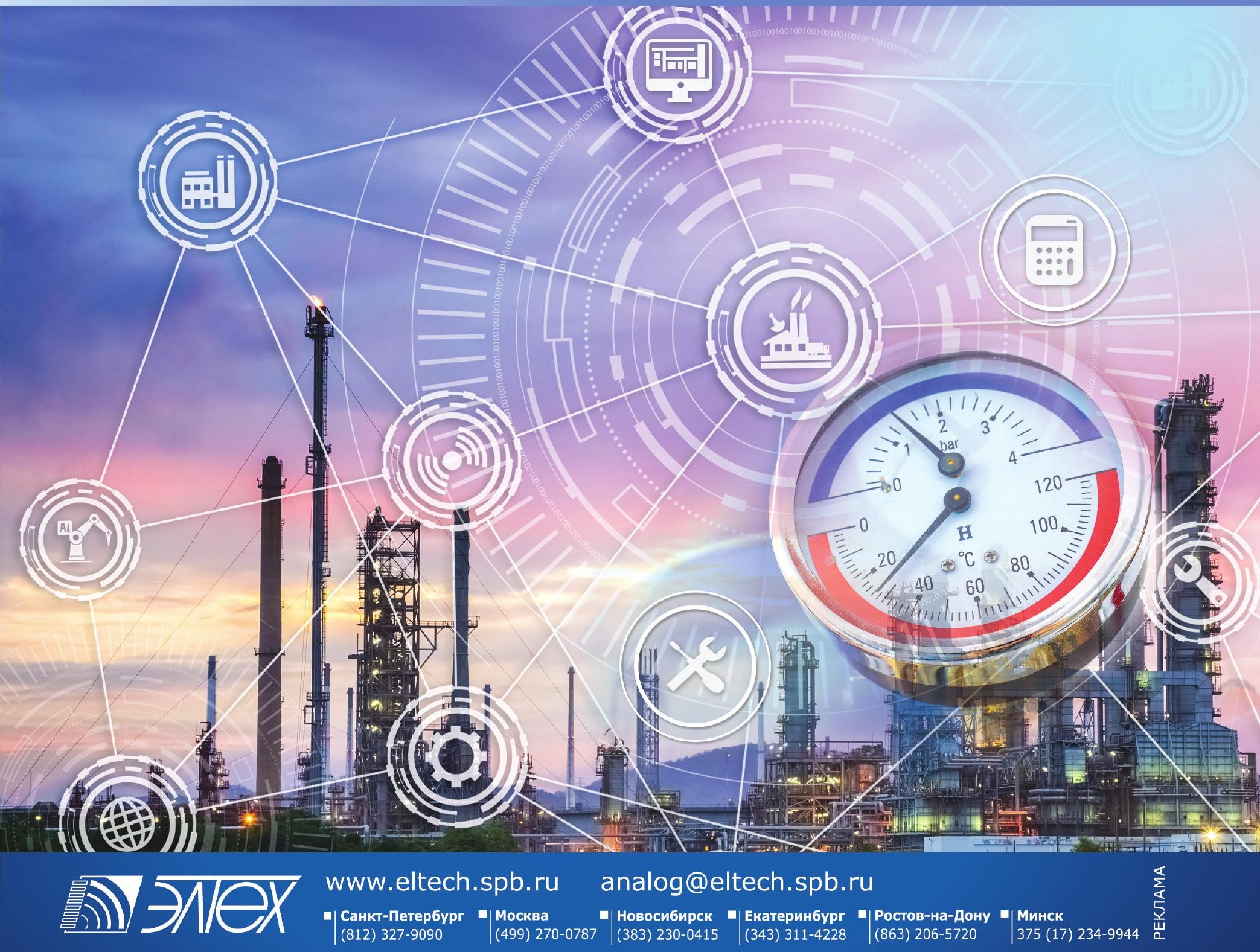

